\title{
Searching for substellar companions of young isolated neutron stars ${ }^{\star}$
}

\author{
B. Posselt ${ }^{1,2, \star \star}$, R. Neuhäuser ${ }^{1}$, and F. Haberl ${ }^{2}$ \\ 1 Astrophysikalisches Institut und Universitäts-Sternwarte, Schillergäßchen 2-3, 07745 Jena, Germany \\ e-mail: bposselt@cfa.harvard.edu \\ 2 Max-Planck-Institut für extraterrestrische Physik, Postfach 131285741 Garching, Germany
}

Received 7 May 2008 / Accepted 27 October 2008

\begin{abstract}
Context. Only two planetary systems orbiting old ms-pulsars have been discovered. Young radio pulsars and radio-quiet neutron stars cannot be analysed by the usually-applied radio-pulse-timing technique. However, finding substellar companions orbiting these neutron stars would be of significant importance: the companion may have had an exotic formation, its observation may also enable us to study neutron-star physics.

Aims. We investigate the closest young neutron stars to Earth to search for orbiting substellar companions.

Methods. Young, thus warm substellar companions are visible in the Near infrared, in which the neutron star itself is much fainter. Four young neutron stars are at sufficient speed to enable a common proper-motion search for substellar companions within few years. Results. For Geminga, RX J0720.4-3125, RX J1856.6-3754, and PSR J1932+1059 we found no comoving companion of masses as low as 12,15, 11, and 42 Jupiter masses, respectively, for assumed ages of 1, 1, 1, and $3.1 \mathrm{Myr}$, and distances of 250, 361, 167, and $361 \mathrm{pc}$, respectively. Near infrared limits are presented for these four and five additional neutron stars for which we have observations for only one epoch.

Conclusions. We conclude that young, isolated neutron stars rarely have brown-dwarf companions.
\end{abstract}

Key words. stars: neutron - stars: pulsars: general — stars: planetary systems - stars: low-mass, brown dwarfs

\section{Introduction}

The presence of substellar companions (planets and brown dwarfs) orbiting neutron stars has been unexpected because the powerful supernova event which produces the compact object. Astonishingly however, the first planetary-mass companions discovered orbiting other stars were neutron star (NS) companions. Three of these objects were detected around the old millisecond(ms)-pulsar PSR 1257+12 (Wolszczan \& Frail 1992; Konacki \& Wolszczan 2003). They have masses as high as four Earth masses and are very close to their primary at distances of between $0.2 \mathrm{AU}$ and $0.5 \mathrm{AU}$. Another planetary system detected orbiting a NS is the binary system of the ms-pulsar PSR B1620-26 and its white dwarf companion (Sigurdsson et al. 2003; Sigurdsson \& Thorsett 2005). A 2.5-Jupiter-mass object orbits the white dwarf and therefore the NS at 23 AU. However, there is no detection of any brown dwarf companion orbiting a neutron star. Several formation models have been discussed to explain the unexpected discovery of NS planets either by assuming supernova survival, by planet formation after the supernova, or by planet capturing (for a detailed review see Podsiadlowski 1995). Planet formation within in-situ discs around NSs could differ from those around solar-like stars. Thus, the comparison of NS planetary systems with those around solar-like stars can be fruitful for understanding planet formation in general. However,

\footnotetext{
* Based on observations made with ESO Telescopes at the La Silla or Paranal Observatories under programme IDs: 66.D-0135, 71.C-0189, 72.C-0051, 74.C-0596, 077.C-0162, 78.C-0686, 79.C-0570.

$\star \star$ Now at the CfA.
}

substellar companions of NSs are interesting not only for planet or brown-dwarf formation theory. They could also provide insights into the supernova event and maybe the composition and final stages of the progenitor star(s). Characterising low-mass companions around NSs, in particular their masses and orbital parameters, can help us to also determine the dynamical masses of the NSs. These masses, together with the stellar radii, constrain the equation-of-state of matter inside the neutron stars. The radius and mass of a neutron star have not been both determined precisely for a given object, and the equation-of-state of ultradense matter in the universe is still unknown. Although the masses of NSs can be derived for binary systems (e.g., Jonker et al. 2003), the most reliable radius estimation comes from the radio-quiet X-ray thermal NSs, which are isolated without stellar companions (e.g., Trümper et al. 2004).

Only two planetary systems have been detected orbiting mspulsars as described above, both by the radio-pulse-timing technique, which measures variations in the pulse-arrival times. This technique is most sensitive to stable ms-pulsars and requires a significant amount of observing time. For ordinary radio pulsars, the timing noise has a significant effect on the precision of the measurements required. Using this method, one is however unable to search for substellar companions orbiting radioquiet neutron stars. The radio-pulse-timing technique is able to detect only relatively close companions, as in radial-velocity planet searches among solar-like stars. Substellar companions in wide orbits cannot therefore be detected with this method. Since NSs are faint at visible wavelengths, the commonly used radialvelocity method or search for astrometric wobbling should 
preferably be done using X-ray data in which many NSs are significantly bright. Unfortunately however, both the spectral and spatial resolutions of X-ray data are prohibitively poor for these methods. Transit searches would be ineffective, because rarely is more than one NS detected in the field of view.

Substellar companions of NSs at any separation can be identified only using their common proper motions measured by direct imaging, primarily in the near infrared (NIR) if the companions are young. They are several magnitudes fainter in the optical. The companions are brighter than the NSs in the NIR, even though lower in mass, because they are larger in size and continue to contract and are, hence, warm when young. Direct detection of close (even separations of a few AU) and faint substellar companions is currently possible only in the vicinity of NSs. Substellar companions in wide orbits can also be detected. However, one is restricted to young objects at small distances from the Sun. The magnitudes of substellar objects (brown dwarfs and giant planets) can be predicted applying the non-gray theory of substellar objects by Burrows et al. (1997) (B97 in the following), who computed IR magnitudes of substellar objects of different age and mass. The upper mass limit of planets is still not yet defined. There are two suggestions: either all substellar objects below the Deuterium-burning limit of about 13 Jupiter masses are referred to as planets, or all objects below the radial-velocity, brown-dwarf desert (of between 20 and 50 Jupiter masses) are called planets, i.e., either all objects below 13 Jupiter masses or below 50 Jupiter masses. Calculation such as those of B97 start with assumed initial conditions about the internal structure and temperature of the substellar objects, and should, hence, be interpreted with care for young objects up to a few Myr (for a discussion see Baraffe et al. 2002). However, we use these calculations for our estimates due to the lack of more suitable ones. According to the calculations of B97, at an age of $100 \mathrm{Myr}$ and a distance of $300 \mathrm{pc}$, a companion at the Deuterium burning mass limit ( $\sim 13 M_{\text {Jup }}$ ) would have an $H$-band magnitude of $22 \mathrm{mag}$, which is easily achieved by current optical telescopes. We present the first results of our search for substellar companions around NSs. We note that some of our data were analysed by Lo Curto et al. (2007). However, they were interested in the spectra of the neutron stars as well as the hypothetical fallback discs around them. They found neither a fallback disc nor that the IR data were of sufficient quality to constrain the physical properties of the NSs from the spectra.

\section{Sample selection}

Although substellar companions may survive a supernova ( $\mathrm{SN})$, this case is unlikely, and the companions probably formed instead after the SN event. Hence, we use the NS age to approximate the age of substellar companions. However, if a companion does indeed survive a SN event, then its age can be significantly underestimated. All NSs younger than $100 \mathrm{Myr}$ and closer than $300 \mathrm{pc}$ were chosen as the initial sample in 2000. The radio pulsars amongst these neutron stars were selected from the pulsar catalogue of Taylor et al. (1993). They were assumed to be ordinary radio pulsars, and still young, since radio emission from non-recycled NSs is expected to last at most $10 \mathrm{Myr}$ (Lorimer \& Kramer 2004). To this sample, the radio-quiet, X-ray thermal, isolated neutron stars (XTINSs) were added. As noted above, the XTINSs are important for constraining the equation of state due to their blackbody-like spectra providing the most reliable radius estimation currently available. They also represent nearly half of the local young neutron star population (for reviews of these NSs, see, e.g., Kaplan 2008; and Haberl 2004).
No close, comoving companions of mass as low as substellar have been identified in deep, optical observations and propermotion studies (e.g., Mignani et al. 2003, Walter \& Matthews 1997; and Motch et al. 2003). The ages of the radio pulsars in our sample are estimated from their characteristic spin-down age, although this characteristic age is a coarse estimation of the true neutron-star age. For the well-known Crab pulsar, the spindown age exceeds the true age by about $25 \%$ (e.g., Manchester \& Taylor 1977). Initally assumed to be younger than $100 \mathrm{Myr}$, some of our selected radio pulsars appear to be older from newer radio-timing data by Hobbs et al. (2004), so that possible substellar companions would be fainter (see individual discussion in Sect. 5). The additional radio-quiet XTINSs, which were detected as soft ROSAT sources, are most probably also young NSs (of <100 Myr): Their proper motions indicate that their origins are most likely in nearby young, star-forming clouds. The XTINSs have strong magnetic fields, an indicator of youth, and inferred spin-down ages from X-ray pulsations of less than 10 Myr (see, e.g., Kaplan 2008). Thus, the XTINSs are probably several Myr old with cooling surfaces that emit X-ray emission. For all seven radio-quiet, X-ray thermal NSs, it appears unlikely that they are old, and that their X-ray emission is due to accretion from the interstellar medium (ISM). First, they have constant X-ray emission over years with one notable exception for which precession of the NS is likely (Haberl et al. 2006). Neuhäuser \& Trümper (1999) also quoted statistical reasons for excluding accretion from the ISM. Furthermore, three radio-quiet, X-ray thermal NSs have known proper motions, which are so high that they imply too low Bondi-Hoyle accretion efficiencies for describing these stars as old $\left(10^{9}\right)$ NSs reheated by accretion from the ISM.

For the radio pulsars, approximate distance estimates are available from the dispersion measure, which indicates the electron column-density towards the pulsar (Taylor et al. 1993; Manchester et al. 2005). For the radio-quiet, X-ray thermal NSs, a parallax measurement is available only in two cases, namely RX J0720.4-3125 and RX J1856.5-3754. For the latter, Walter \& Lattimer (2002) determined a distance of $\sim 117 \mathrm{pc}$ with the Hubble Space Telescope. Kaplan et al. (2007) reported another, preliminary distance estimation with RX J1856.5-3754 of $167_{-15}^{+18}$ pc. For RX J0720.4-3125 Kaplan et al. (2007) reported a parallactic distance of $361_{-88}^{+172} \mathrm{pc}$. The measured column densities from the X-ray spectra of the other XTINSs are similar to those of RX J0720.4-3125 and RX J1856.5-3754, all smaller than the Galactic values (towards the respective direction of sight). One can assume the same order of magnitude for their distances as for RX J1856.5-3754 and RX J0720.4-3125. Taking into account absorption by the inhomogenously distributed ISM, the estimated distances for two further radio-quiet X-ray thermal NSs support this assumption (Posselt et al. 2007). Here, we consider $300 \mathrm{pc}$ to be the lower distance limit for the other 5 XTINSs.

In the following we will present the observations and results for nine neutron stars, four of them observed at two epochs. All objects, their known or assumed distances, and their proper motions are listed in Table 1.

Given the age and distance $d$ of the NSs, the expected $H$-band magnitude at which we should find brown dwarfs and massive gas planets can be derived by applying the results of B97. Bolometric corrections in the $K$-band have a reported range from $\approx 2$ (T5 dwarf) to $\approx 3.5$ (L5 dwarf) (Golimowski et al. 2004). In the following we use a bolometric correction of $\mathrm{BC}=\mathrm{BC}_{\mathrm{H}} \simeq$ $\mathrm{BC}_{K}=3.5 \mathrm{mag}$. 
Table 1. The observed neutron stars, their distances, and proper motions.

\begin{tabular}{lccc}
\hline \hline Object & $\begin{array}{c}D \\
{[\mathrm{pc}]}\end{array}$ & $\begin{array}{c}\mu_{\alpha} \\
{\left[\mathrm{mas} \mathrm{yr}^{-1}\right]}\end{array}$ & $\begin{array}{c}\mu_{\delta} \\
{\left[\mathrm{mas} \mathrm{yr}^{-1}\right]}\end{array}$ \\
\hline PSR J0108-1431 & $130^{a}$ & $<26^{a}$ & $<78^{a}$ \\
RX J0420.0-5022 & $\geq 300^{a s s}$ & $<123^{b}$ & $<123^{b}$ \\
Geminga & $250_{-62}^{+120 c}$ & $142.2 \pm 1.2^{c}$ & $107.4 \pm 1.2^{c}$ \\
RX J0720.4-3125 & $361_{-88}^{+172 d}$ & $-93 \pm 1.2^{d}$ & $52.8 \pm 1.3^{d}$ \\
RX J0806.4-4123 & $240^{a s s}$ & $<86^{b}$ & $<86^{b}$ \\
RX J1856.6-3754 & $167_{-15}^{+18 d}$ & $326.7 \pm 0.8^{e}$ & $-59.1 \pm 0.7^{e}$ \\
PSR J1932+1059 & $361_{-8}^{+10 f}$ & $94.03 \pm 0.14^{f}$ & $43.37 \pm 0.29^{f}$ \\
PSR J2124-3358 & $270 \pm 20^{g}$ & $-14.4 \pm 0.8^{h}$ & $-50 \pm 2^{h}$ \\
RBS 1774 & $\geq 300^{\text {ass }}$ & not known & not known \\
\hline
\end{tabular}

${ }^{K} 1$ RXS J214303.7+065419; ${ }^{A}$ PSR J2124-3358 turned out to be too old for our substellar companion search. See Appendix C for a NIR limit of this neutron star; ${ }^{a}$ Mignani et al. (2003); ${ }^{b} 2 \sigma$ limit of Motch et al. (2009); ${ }^{c}$ Faherty et al. (2007); ${ }^{d}$ Kaplan et al. (2007); ${ }^{e}$ Walter \& Lattimer (2002); ${ }^{f}$ Chatterjee et al. (2004); ${ }^{g}$ Gaensler et al. (2002); ${ }^{h}$ Hotan et al. (2006); ${ }^{a s s}$ notes an assumed distance, which is a lower limit as indicated by estimations from X-ray measured hydrogen column density (e.g. Posselt et al. 2007), see also text.

\section{Observation summary and data reduction}

\subsection{First epoch observations}

Nine neutron stars were observed in the $H$-band utilising the Very Large Telescope (VLT) equipped with the Infrared Spectrometer And Array Camera (ISAAC) in service mode. The objects and the details of observations are listed in both Table 1 and Table A.1 in Appendix A.

The VLT observation durations ranged between two and three hours. The seeing was usually superior to $0.8^{\prime \prime}$, and the airmass in most cases was below 1.5. The jitter-Mode was used with individual exposures of $12 \mathrm{~s}$ and a jitter box width of $20^{\prime \prime}$. The data reduction was completed using ESO's Eclipse (version 4.8.1 and 5.0.) (Devillard 1999, 2001), taking into account the corresponding darks, sky flats and bad pixel maps obtained during ESO's regular calibration plan.

Individual images with poor seeing or high airmass have been excluded. For comparison, ESO's ISAAC pipeline has also been applied. Multiple observations of the same object were reduced individually. The results were then combined using IRAF (Tody 1986, 1993) if observing conditions were similar. We completed the absolute astrometry for the first epoch observation with the 2MASS point-source catalogue (PSC, Skrutskie et al. 2006) using the Graphical Astronomy and Image Analysis Tool (GAIA) (Draper et al. 2007). For many relatively bright 2MASS sources in the field (e.g. RX J1856.6-3754), a positional accuracy of 300 mas ( $3 \sigma$, Skrutskie et al. 2006) can be achieved. In some fields, only a handful of 2MASS sources are present. In these cases, we used the USNO B1 catalogue for which Monet et al. (2003) stated an astrometric uncertainty of 200 mas for absolute astrometry. However, this optical catalogue is based on far older observations than 2MASS, and its entries may not correspond to apparently matching NIR detections in our observations. We use absolute astrometry only in deriving NIR limits for the neutron-star position, taking an appropriate error circle in considering astrometric calibration effects as well as the proper motions of the neutron stars. Neutron star positions are taken to be either a VLBI position (e.g., PSR J1932+1059) or the position of the optical counterpart (e.g., RX J1856.6-3754), or, if those are not known, the X-ray position (e.g., RBS 1774). References are either the same as for the proper motions in Table 1, or they are given in the individual introductions of Sects. 5.1.1 to 5.2. The NS's positional uncertainties have no impact on our search for substellar companions; in Sect. 5.3, appropriate sky regions are considered in searching for a possible NIR counterpart to a NS.

No standard stars were explicitely observed for this project since only rough photometry was needed. In completing our photometric calibration, we applied the zeropoints obtained by ESO during their regular ISAAC calibration plan as a starting point, and calibrated further with 2MASS photometry by using - preferably high quality - 2MASS point sources in the field (for individual details see Sect. 5).

\subsection{Second epoch observations}

All second epoch observations (see Table A.1) were also carried out in the $H$-band in a similar jitter mode as the first epoch observations. The second epoch observations of RX J1856.6-3754 and PSR J1932+1059 in May 2006 were shifted to the $3.5 \mathrm{~m} \mathrm{New}$ Technology Telescope (NTT) equipped with the Son of ISAAC instrument (SOFI) (instead of VLT ISAAC). The two fields were observed with SOFI's Small field objective for around three to four hours during each of the four nights. The seeing was usually of higher quality than $1^{\prime \prime}$, and during the second and third night most of the time superior to $0.8^{\prime \prime}$, and in the last night variable. The observations were reduced by applying the SOFI pipeline procedures at the telescope. We cross-checked the result by reducing individual run data additionally with Eclipse. The further data-reduction procedure was the same as for the first epoch observations applying IRAF and GAIA. The second epoch SOFI observations were up to 1 mag less deep than the ISAAC observations.

In May 2007, VLT NAos COnica (NACO) observations were completed for the inner field around PSR J1932+1059 using the coronographic mode for a bright star close to the pulsar. NACO is an adaptive optical (AO) imager and spectrometer and has been operated with the S54 camera. The Visual dichronic element and wavefront sensor $(0.45-1.0 \mu \mathrm{m})$, and the opaque $1.4^{\prime \prime}$ corongraph were used. A jitter box of $20^{\prime \prime}$, coronographic individual exposures of $60 \mathrm{~s}$, sky individual exposures of $0.3454 \mathrm{~s}, 4$ "object" offset positions and 6 "sky" offset positions were further parameters of this NACO run. Observing conditions for the approximately one-hour observation were very good with seeing superior to 0.6 at an airmass of around 1.3 and a strehl ratio of around $17 \%$. The NACO observations were reduced by applying the jitter engine of Eclipse (for more details, see Appendix A).

Second epoch observations of RX J0720.4-3125 and Geminga were obtained with VLT ISAAC in December 2006 and January/February 2007 respectively (for more details see Table A.1). The data reduction procedure was the same as for the first epoch ISAAC observations applying Eclipse, ISAAC pipeline, IRAF, and GAIA.

The NACO field of view is $56^{\prime \prime} \times 56^{\prime \prime}$ and therefore smaller than those of ISAAC and SOFI with $154^{\prime \prime} \times 154^{\prime \prime}$. The (nominal) pixel sizes are $0.054^{\prime \prime}$ per pixel for NACO, $0.148^{\prime \prime}$ per pixel for $I S A A C$, and $0.144^{\prime \prime}$ per pixel for SOFI.

\section{Method}

From the known proper motions of the neutron stars, it is possible to calculate the expected shifts in pixel coordinates. First 
Table 2. Observational parameters for neutron stars with two epochs of observations.

\begin{tabular}{|c|c|c|c|c|c|c|c|c|c|}
\hline Neutron star & $\begin{array}{l}m_{\mathrm{BD}}{ }^{a} \\
{[\mathrm{mag}]}\end{array}$ & $\begin{array}{l}m_{13 \mathrm{Jup}}{ }^{a} \\
{[\mathrm{mag}]}\end{array}$ & $\begin{array}{l}m_{\lim }{ }^{b} \\
{[\mathrm{mag}]}\end{array}$ & $\begin{array}{c}\text { Epoch difference } \\
{[\mathrm{yr}]}\end{array}$ & $\begin{array}{l}r_{\text {proj }}{ }^{c} \\
{[\mathrm{AU}]}\end{array}$ & $\begin{array}{l}\mathrm{refX}^{d} \\
{[\mathrm{mas}]}\end{array}$ & $\begin{array}{l}\mathrm{refY}^{d} \\
{[\mathrm{mas}]}\end{array}$ & $\begin{array}{c}\text { Align }_{X}^{e} \\
+/- \\
\end{array}$ & $\begin{array}{c}\text { Align }_{Y}{ }^{e} \\
+/-\end{array}$ \\
\hline Geminga & 18.2 & 22.3 & 22.4 & 3.05 & 10000 & 148.4 & 148.4 & + & - \\
\hline RX J072 & 19.0 & 23.0 & 22.9 & 2.95 & 20000 & 147.7 & 147.7 & + & - \\
\hline RX J1856.6-3754 & 17.3 & 21.4 & 21.5 & 3.0 & 10800 & 147.8 & 147.7 & + & + \\
\hline PSR J1932+1059 & 21.5 & 23.5 & 21.5 & $2.9,3.9$ & see text & 147.5 & 147.3 & - & + \\
\hline
\end{tabular}

${ }^{a} m_{\mathrm{BD}}$ and $m_{13 \mathrm{Jup}}$ are the brightest $H$-band magnitudes expected for a brown dwarf companion or a gas planet of 13 Jupiter mass respectively (see also text); ${ }^{b} m_{\text {lim }}$ is the magnitude limit reached in both epochs; ${ }^{c} r_{\text {proj }}$ is the covered projected physical separations; ${ }^{d}$ (refX, refY) are the pixel scale of the reference $I S A A C$ image; ${ }^{e}$ reference image axes are roughly aligned with RA (X) and DEC (Y) to within 1 degree, although sometimes with opposite direction (indicated by "-" instead of "+").

and second epoch observations have to be compared regarding object detections, magnitudes, and positions. Due to the chosen time span, it should be possible to differentiate between the expected shifts for comoving objects and position errors. We tested several source detection algorithms, e.g. the Source Extractor by Bertin \& Arnouts (1996), to determine the most reliable relative astrometry. We refer to Sect. B for more details. We concentrate on our final approach for the relative astrometry, the StarFinder-Code by Diolaiti et al. (2000b), an IDL-based point source detection algorithm for crowded fields, which begins by determining the point spread function (PSF) from the science image for further fitting. The performance of StarFinder was tested, e.g., by Diolaiti et al. (2000a) and Christou et al. (2004). The latter found, for example, that in fields with overlapping stellar PSFs StarFinder was capable of producing consistent astrometry of accuracy corresponding to one tenth of the PSF or higher. Photometric errors are usually smaller than about $0.1 \mathrm{mag}$ (Diolaiti et al. 2000a; Christou et al. 2004). For extremely faint sources, StarFinder is sensitive to the structures in the determined model PSF. We carefully selected the reference sources for the model PSF and noise estimation. Only objects detected with a signal-to-noise ratio (SNR) of at least three in both epochs - ISAAC, SOFI and NACO observations - were considered in the search for comoving objects. The correlation factor for the StarFinder detections were usually larger than 0.7. All sources were manually inspected and apparent galaxies or artefacts were excluded. If we compared sources detected by two different instruments, then the (SOFI, NACO) pixel coordinates were converted to coordinates within the ISAAC reference frame applying WCStools (Mink 2002). Source lists of two epochs were then correlated, and we derived the positional shifts presented in Sect. 5. For the two epochs of ISAAC observations, we minimised systematic, instrument-related errors such as field distortions by a similar observation setup. If different instruments/telescopes were used, the additional systematic errors showed up in a broader scattering of the computed positional shifts. For the NACO observations, this extra error was neglible for the chosen ISAAC reference frame.

If no second epoch observation was obtained, e.g., due to still unknown proper motion, we investigated the NIR sources in a $5000 \mathrm{AU}$ circle about the NS position for their opticalNIR colours to identify potentially interesting objects for followup observations. We refer to Sect. B.2 for more details of the procedure.

At the positions of the NSs, we also searched for faint NIRobjects, which could indicate unexpected possible counterparts of the NSs themselves. We determined an upper $H$-band limit for the NS if no NIR object was found within the area defined by the positional error, by taking the faintest, close-by object of a SNR higher than 3 . We checked whether we could reach deeper NIR-limits at the position of the NSs by combining first and second epoch observations.

\section{Results}

\subsection{Pixel shifts between two epochs of rapidly moving neutron stars}

All faint NIR sources close to the known NS position were analysed as described in Sect. 4. Comoving companion candidates, identified by comparing first and second epoch observations, would have a high probability of being true companions, because NSs have large proper motions. Therefore, it would be unlikely that an unrelated, distant, faint background object would share such a large proper motion.

Table 2 lists some parameters of the analysis. Additional notes on these and the results can be found in Sects. 5.1.1-5.1.4.

\subsubsection{Geminga}

Geminga is the prototype $\gamma$-ray pulsar (see e.g. Bignami \& Caraveo 1996). The distance of Geminga is still under discussion. Faherty et al. (2007) estimated its parallactic distance to be $250_{-62}^{+120} \mathrm{pc}$, in contrast to the older value of $157_{-34}^{+59} \mathrm{pc}$ derived by Caraveo et al. $(1996,1998)$. We adopt in the following the distance measured by Faherty et al. (2007). Geminga has a spin-down age of around 340000 years. The lowest age considered by B97 (or similar papers, e.g., Baraffe et al. 1998, 2002) was 1 Myr. We consider 383 sources in the entire ISAAC field of Geminga, most of them fainter than $18.2 \mathrm{mag}$. The pixel shifts of the objects, common to both epochs, are plotted in Fig. 1. There is no apparent comoving source within a distance of $\approx 10000 \mathrm{AU}$ from Geminga to magnitudes as faint as $H=22.4$ mag. For a $1 \mathrm{Myr}$ age (see above) and $250 \mathrm{pc}$, this correponds to an exclusion of all young companions of more massive than 12 Jupiter masses following the calculations of B97.

\subsubsection{RX J0720.4-3125}

RX J0720.4-3125 is the second brightest of the X-ray thermal, isolated neutron stars. We assume a similar age of $1 \mathrm{Myr}$ to that of RX J1856.6-3754. The distance to RX J0720.4-3125 is still of large uncertainty. Kaplan et al. (2007) reported a parallactic distance from optical observations of $361_{-88}^{+172} \mathrm{pc}$. The hydrogen column density fitted to the $\mathrm{X}$-ray spectrum indicates a distance of between 240 and 270 pc, taking into account the local imhomogenously distributed interstellar medium (Posselt et al. 2007). In the second epoch, we had to reduce the correlation coefficient contraint to 0.65 to reidentify all first epoch detections. Fourteen sources (out of the considered 581) in the second 

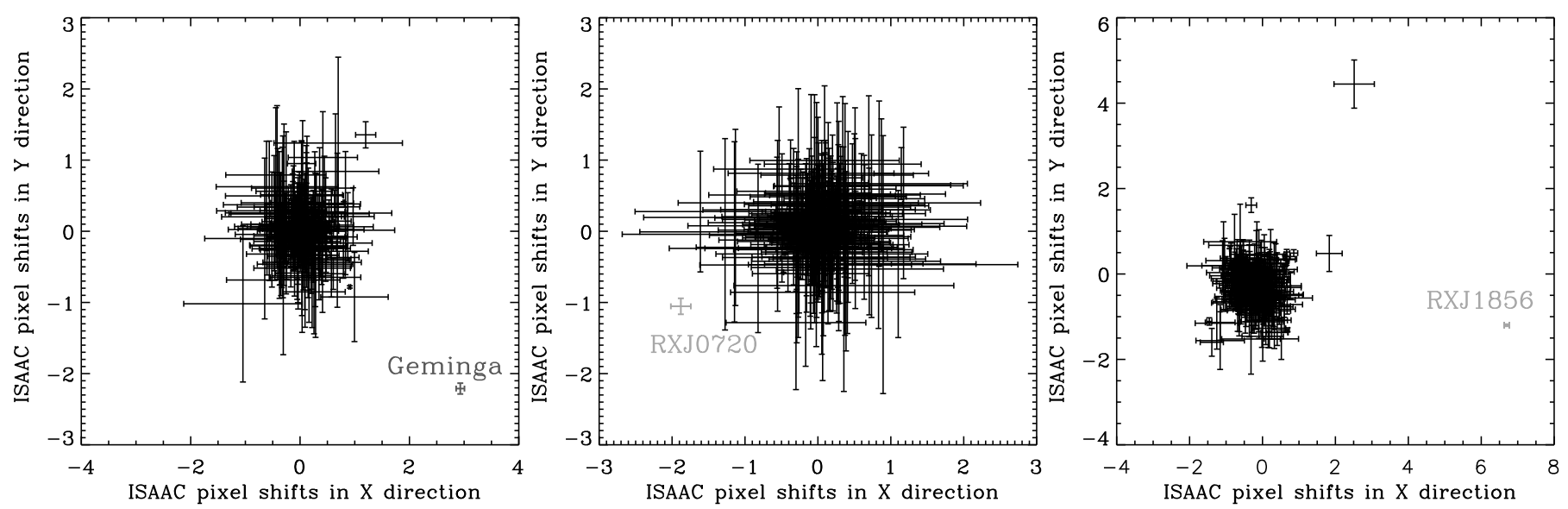

Fig. 1. Pixel shifts between the first and second epoch NIR observations of Geminga, RX J0720.4-3125, and RX J1856.6-3754 (from left to right). Errors are $3 \sigma$-StarFinder positional errors. The grey positions marked Geminga, RXJ0720, and RXJ1856 show the positions a possible comoving object would have. For more details regarding the ISAAC reference frame pixel scale, axes orientation, and covered magnitudes see Table 2 as well as Sects. 5.1.1-5.1.3.

epoch have correlation coefficients of between 0.65 and 0.7 . The pixel shifts of the objects common to both epochs are plotted in Fig. 1. There is no apparent comoving source within $\approx 20000 \mathrm{AU}$ of RX J0720.4-3125 as faint as $H=22.9$ mag. For a 1 Myr age and $361 \mathrm{pc}$ distance, this correponds to an exclusion of all young companions more massive than 15 Jupiter masses following the calculations of B97.

\subsubsection{RX J1856.6-3754}

RX J1856.6-3754 is the brightest of the X-ray thermal neutron stars. Its $H S T$-determined parallax of $117 \pm 12$ pc reported by Walter \& Lattimer (2002), was revised to $142_{-39}^{+58} \mathrm{pc}$ by Kaplan et al. (2002) and revised further to a preliminary value of $167_{-15}^{+18}$ pc by Kaplan et al. (2007). In the following, we adopt this most recent value. The age of RX J1856.6-3754 is assumed to be around $1 \mathrm{Myr}$ given the proper motion and likely birth place in Upp Sco (e.g. Walter \& Lattimer 2002). There are 525 common objects of SNR higher than three in both, the ISAAC and SOFI observations, 448 of them fainter than $H=17.3 \mathrm{mag}$, and two with $H \geq 21.4$ mag. In Fig. 1, we plot both the pixel shifts between the two epochs and the pixel shift of the neutron star, which would be identical for a comoving object. We accounted neither for pixel-scale errors or instrumental-distortion errors. Both are present in the scatter distribution of the object's pixel shifts. We do not expect an additional positional error larger than one fifth pixel (corresponding to the mean $3 \sigma$ positional uncertainty) from coordinate transformation (SOFI to $I S A A C$ reference frame). Even taking this into account does not alter the trend observed in Fig. 1 - there is no comoving $H$-band source of $S N R \geq 3$ present in our observations. Applying the calculations of B97 for an assumed age of 1 Myr and a distance of $167 \mathrm{pc}$, substellar companions heavier than $0.01 M_{\odot}$ or 10.5 Jupiter masses can be excluded from existing around RX J1856.6-3754.

We include a short note about the two objects with exceptional pixel shifts in the right-hand panel of Fig. 1. The object with offset of about $(1.8,0.5)$ belongs to a triple ISAAC source. It has an ISAAC $H$-band magnitude of $20.6 \mathrm{mag}$, the other two sources have $H=19.6 \mathrm{mag}$ and $21.3 \mathrm{mag}$, and are located roughly $1.2^{\prime \prime}$ from each other. In the SOFI data, these three sources are less well resolved, and StarFinder can detect only two applying selections of $S N R \geq 3$ and correlation coefficient $\geq 0.7$. The derived second epoch position of the object in question is shifted slightly towards the position of the undetected fainter object. There were around ten such cases of nondetected multiple sources in this particular field. Usually, the positions derived by StarFinder are only marginally influenced. The pixel shift of around $(2.5,4.4)$ belongs to an 21.0 mag object that is offset in position by about $1.6^{\prime \prime}$ from a 16.5 mag object. By eye inspection, this source is hardly visible in the SOFI image. StarFinder succeeds in detecting the faint source, although its position appears be unreliably determined for such a high contrast.

\subsubsection{PSR J1932+1059}

PSR J1932+1059 is a radio pulsar. New VLBI measurements place this neutron star at a distance of $361 \pm 10$ pc (Chatterjee et al. 2004), further away than previous estimates. Therefore, only brown dwarfs could be detected as possible substellar companions, given its spin-down age of $3.1 \times 10^{6}$ years (Hobbs et al. 2004), and following the models by B97. Unfortunately, PSR J1932+1059 is located in a crowded field that includes a bright, $H=8.8 \mathrm{mag}$, object only $\approx 2.5^{\prime \prime}$ away from the pulsar. The sources visible in the image of the combined SOFI observations of May 2006 were less sharply resolved than those of the ISAAC data due to the observing conditions (small visibility time window, and not so good weather on the last night). The bright NIR object causes large "doughnut"-shaped artefacts (only in the SOFI image) due to the jittering process. While the StarFinderCode is able to detect the NIR sources, we were able to avoid most of the artefacts and the bright source by using the NACO coronographic observations from May 2007. We note that the NACO S54 camera has a pixel scale of around 54 mas compared to around 148 mas for ISAAC. NACO StarFinder positional errors are far smaller than the positional errors for ISAAC. NIR sources within an area defined by an approximate $10000 \mathrm{AU}$ projected physical separation of the pulsar (at an assumed distance of $361 \mathrm{pc}$ ) were considered for further analysis. The area 

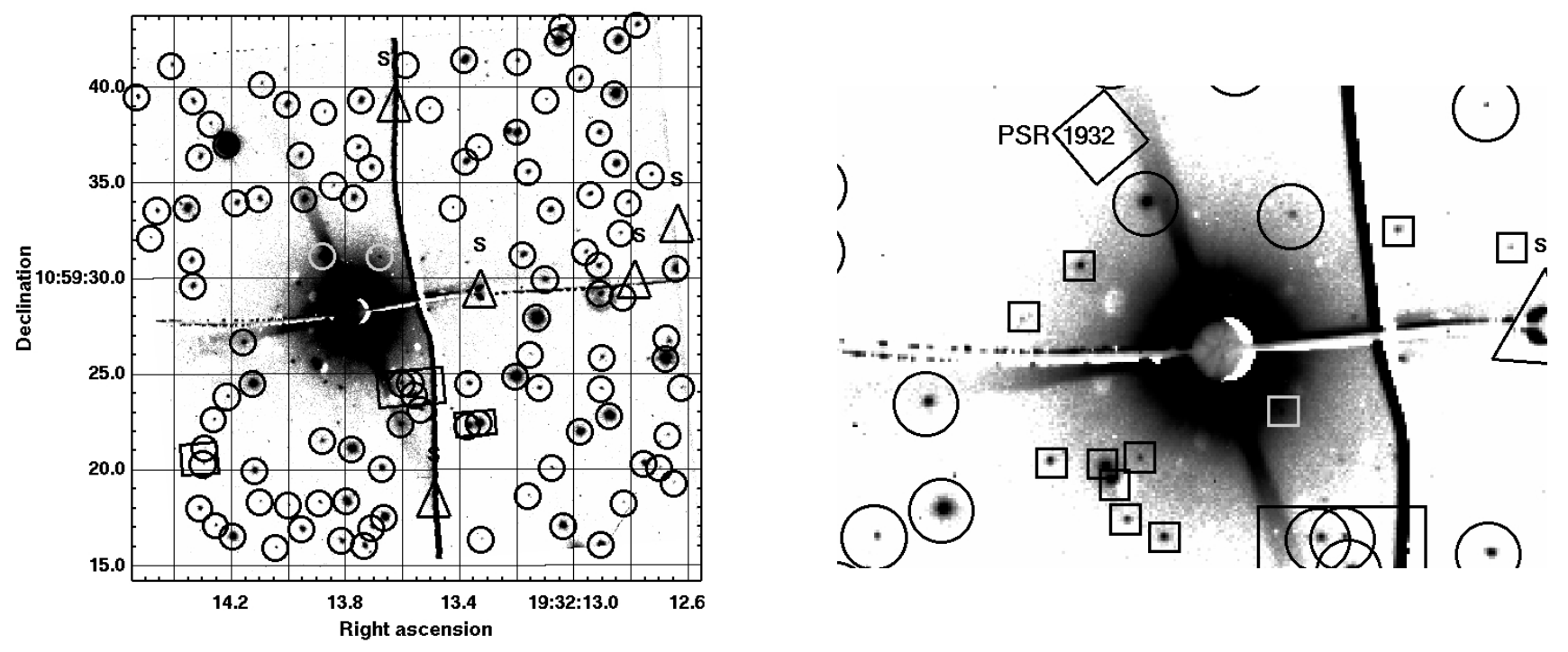

Fig. 2. The NACO-field of PSR J1932+1059 with circles indicating sources common to both NACO and ISAAC observations. The large boxes point toward multiple $N A C O$ detections that were identified to be only one source in the ISAAC image. Triangles with "S" indicate positions of sources for which we used the SOFI observation for the common proper-motion search. The right figure is a zoomed-in (roughly 14 " $\times 10^{\prime \prime}$ ) variant. Sources not considered for the common proper-motion search are marked with squares. They are detected only in the NACO image due to the coronographic mode, but not in the ISAAC observation. The (empty) position of the pulsar is indicated by the diamond shape with $1^{\prime \prime}$ radius.

covered by $N A C O$ extends to the east and north by up to about $3000 \mathrm{AU}$, and to the west and south by up to projected physical separations of roughly $6500 \mathrm{AU}$ from the NS. In this area, we used the SOFI image for only four sources, which were not detected well in the NACO image because of the wire construction shadow. Due to the coronographic NACO AO mode and a smaller pixel scale, more sources were detected than in the ISAAC image. By checking the NIR sources found in both epochs, several single $I S A A C$ sources were found to be multiple NACO sources. StarFinder positional errors for the ISAAC sources were not significantly high in these cases, which indicates the limits of StarFinder. For a relatively bright main component in the multiple source, it was reasonable to assume that this specific source was detected in the ISAAC image. If the multiple $N A C O$ sources were of almost identical magnitude, we compared the flux-weighted average position of the individual $N A C O$ sources with the only one ISAAC position. Furthermore, we considered the pixel shift of each individual $N A C O$ source relative to its $I S A A C$ source to determine how significant the pixel shifts could be due to unidentified, multiple sources. All considered common NACO-ISAAC sources are marked in Fig. 2. Close $\left(<\approx 4^{\prime \prime}\right)$ to the bright star, there are eleven NACO sources that were not detected by ISAAC at all. These sources have $H$ band magnitudes of between 18 and 21 mag. They are indicated in Fig. 2 and should be investigated for possible comotion using data for another epoch.

The pixel shifts of the 284 sources considered for the common proper-motion search in the SOFI and the ISAAC observations are plotted in Fig. 3. Due to the observing conditions, the errors in the SOFI observations are relatively large. There is a faint 21.2 mag object labeled "X1" in Fig. 3 (left), whose $3 \sigma$ error bars overlap with the position of an object that could share a common proper motion with PSR J1932+1059. The spread in the pixel shifts in Fig. 3, especially the fragmentation into two "clouds" indicate a systematic effect. The SOFI small field is known to be strongly affected by distortions in some parts of the instrument field of view, which could explain X1's position in the pixel shift plot. To correct for the distortions, we applied SCAMP (Bertin 2006) with a polynom degree of five, to both the ISAAC and SOFI images. As expected, there were significant SOFI distortions, while for ISAAC distortions were small. We then applied $S W a r p^{1}$ by Bertin (Terapix project) to resample our data, and proceeded with StarFinder for a similar analysis as described above.

The resulting pixel shift plot is shown in Fig. 3 (center). The sources are far more clustered in this figure. Sources with large pixel shifts are either moving objects or they are close to bright stars, which can affect their detection parameters. The X1-object and the pulsar proper motion do not coincide to within their $3 \sigma$ uncertainties, although only marginally. It is likely that X1 is a background object. If, unexpectedly, $\mathrm{X} 1$ is a comoving companion, this source would be at a projected physical separation from the pulsar of around $9200 \mathrm{AU}$, and applying the calculations by B97, it would have a mass of around 42 Jupiter masses for a distance of $361 \mathrm{pc}$ and an age of $3.1 \times 10^{6}$ years. There is no known object within $10^{\prime \prime}$ of $\mathrm{X} 1$ in the SIMBAD-database. Unfortunately, X1 was not in the field of view of our NACO observations.

The pixel shifts of the 104 NACO sources with respect to the earlier ISAAC observations are shown in Fig. 3. The NACO sources "I a" and "I b" belong to one 17.2 mag ISAAC detection, and "II a" - "II c" to another detection of $19.1 \mathrm{mag}$. It appears likely that the single ISAAC sources are a merger of the multiple $N A C O$ detections. The flux-weighted average $N A C O$ positions can be regarded as the putative ISAAC positions if this instrument had been used. The respective flux-weighted average pixel shifts "I weigh" and "II weigh" are situated within the bulk of the pixel shifts of the other sources. Therefore, the ISAAC sources "I" and "II" can be excluded from being comoving objects. The large pixel shifts amongst I a-b, II a-c (each NACO source considered separately) demonstrate that analysing detections by two different instruments with data of different spatial resolution is of limited use given the high precision required to complete the relative astrometry. Thus, to detect the co-motion of all individual $N A C O$ sources with sufficient accuracy, data from an additional $N A C O$ epoch would be required. Overall, Fig. 3 shows for the

${ }^{1}$ http://terapix $\cdot$ iap.fr/rubrique $\cdot$ php?id_rubrique $=49$ 

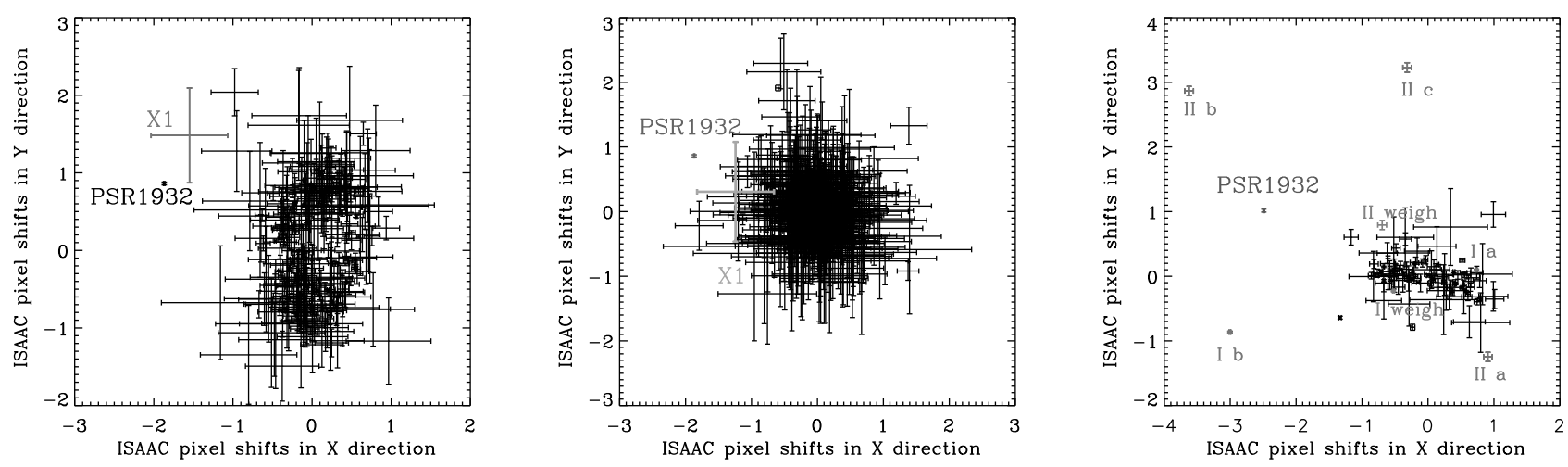

Fig. 3. Pixel shifts between the first and second epoch NIR observations of PSR J1932+1059. Errors are $3 \sigma-S t a r F i n d e r$ positional errors. "PSR1932" marks the position of an object that would comove with PSR J1932+1059, the shown error bars correspond to the $3 \sigma$ uncertainty in the pulsars proper motion. The left plot is for a distortion-uncorrected analysis of the ISAAC and SOFI images. The 21.2 mag object labeled "X1" is 25.6 " north-east of the pulsar. The middle plot shows the pixel shifts of the same objects, but for distortion-corrected ISAAC and SOFI images. The resampled ISAAC pixel scale is 148.4 mas for $x$, and 148.4 mas for $y$. The object X1 and the pulsar proper motion do marginally not coincide within their $3 \sigma$ uncertainties. Pixel shifts for common sources in the ISAAC and the NACO observation are shown in the right plot. As described in the text, single ISAAC sources were found to be multiple NACO sources. Pixel shifts of the two components of the ISAAC source "I" and the three components of the ISAAC source "II" are labeled in the plot as well as the flux-weighted average positions of Ia,b and IIa-c. See Table 2 and text for more details.
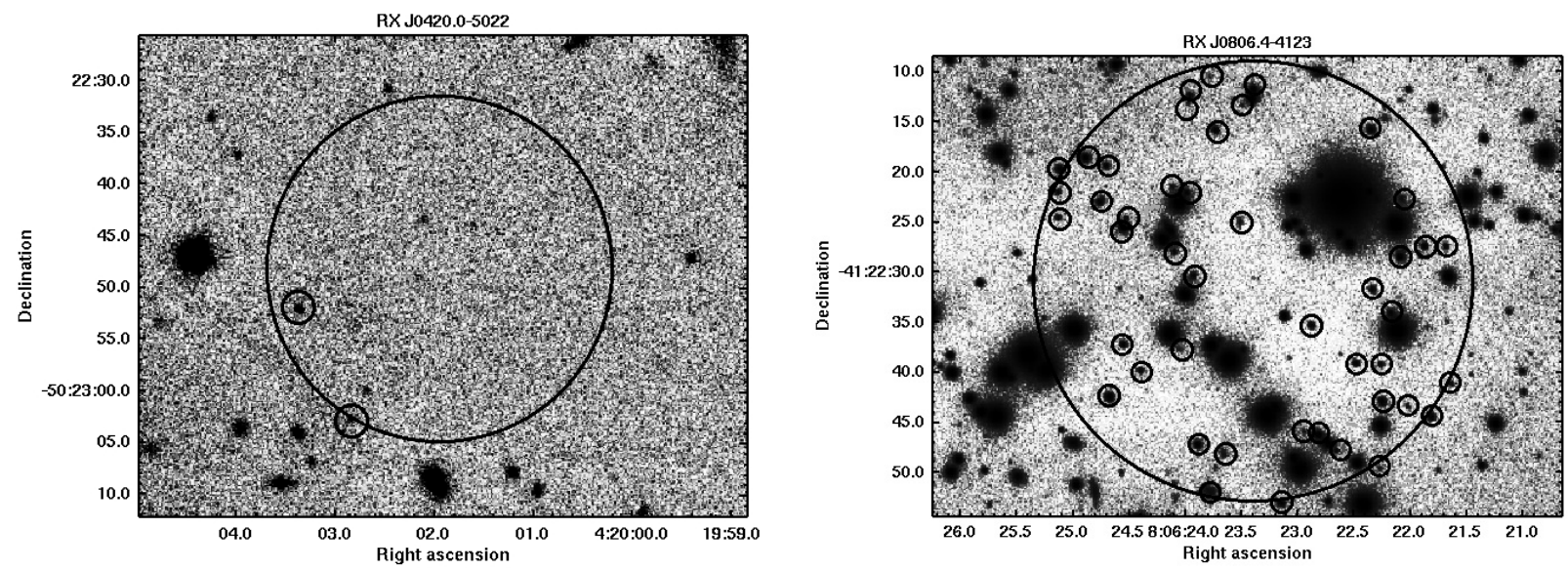

Fig. 4. Faint NIR-objects around RX J0420.0-5022 and RX J0806.4-4123 that may be interesting candidates for second epoch observations. The large circles have a radius of $5000 \mathrm{AU}$ at the respective distances. The marked faint objects have a detection SNR of at least three.

common NACO-ISAAC sources no pixel shifts of the order of that would be expected from a comoving object, although another NACO observation would be needed to confirm this.

The current state of our search around PSR J1932+1054 is that we have found no comoving object brighter than $H=$ 21.5 mag by comparing ISAAC with SOFI observations, as well as ISAAC with NACO observations. At the given distance and age, there are probably no substellar companions more massive than 42 Jupiter masses. However, this result must be seen in the light of the described uncertainties due to the different instruments, and the close-by, bright star. Further investigations are needed for confirmation.

\subsection{Neutron stars with only first epoch observations}

In the following, the first epoch $H$-band observations and available optical data are considered in assessing the necessity of acquiring second-epoch ISAAC observations.
PSR J0108-1431 is one of the closest radio pulsars according to its low dispersion measure $\left(2.38 \pm 0.01 \mathrm{~cm}^{-3} \mathrm{pc}\right.$, D'Amico et al. 1998). The faint pulsar has a distance of $130 \mathrm{pc}$ and appears to be older (170 Myr, Mignani et al. 2003) than we initially assumed. Possible brown dwarfs around PSR J0108-1431 are expected to be fainter than $H=20.8 \mathrm{mag}$. The measured photometric magnitudes for the objects of interest have large error bars due to the availability of only two bright 2MASS sources for the zeropoint determination. Astrometric positions were refined using USNO B1 point source positions. Within $5000 \mathrm{AU}$, there is only one source fainter than $H=20.8 \mathrm{mag}$ having $H=21.0 \mathrm{mag}$. We obtained archival VLT FORS $1 \mathrm{im}-$ ages in the $V$-band, completed the data reduction including bias subtraction and flat-fielding. and calibrated the source $V$-band magnitudes using the tabulated values of Mignani et al. (2003). The faint NIR-object is detected in the $V$-band at a magnitude of $25.6 \mathrm{mag}$, and the $V-H$-colour is therefore $4.6 \mathrm{mag}$. This colour is typical of spectral type M2 V or M3 V, according to Kenyon \& Hartmann (1995). Even when taking into account an error of 1 mag the source is not a candidate for a substellar companion around PSR J0108-1431. Thus, from our VLT ISAAC image 


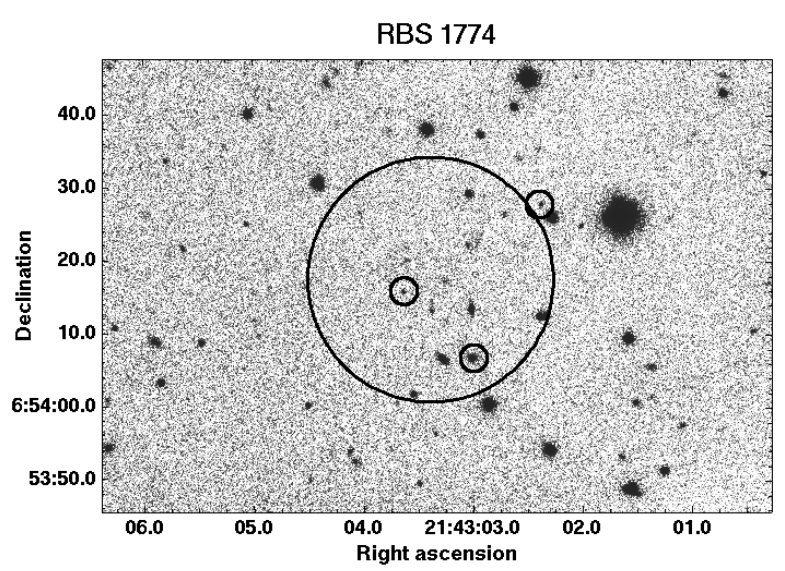

Fig. 5. Faint NIR-objects around RXS J214303.7+065419 that may be interesting for second epoch observations. The large circle would have a radius of $5000 \mathrm{AU}$ at $300 \mathrm{pc}$. The marked faint objects have a detection SNR of at least three.

within 5000 AU of the location of PSR J0108-1431, there are no substellar companion candidates for which second-epoch observations should be obtained.

$\mathrm{RX}$ J0420.0-5022 is a X-ray thermal isolated neutron star (e.g. Haberl et al. 2004). Neither distance nor proper motion have been measured due to a still uncertain optical counterpart. The hydrogen column density fitting the X-ray spectrum indicates a distance of approximately $300 \mathrm{pc}$. Assuming a similar age to that of the more well known XTINS RX J1856.6-3754, one would expect substellar companions to be fainter than $H=19 \mathrm{mag}$ at this distance. Only two objects are present within 5000 AU of the neutron star position at an assumed distance of $300 \mathrm{pc}$. Optical archival observations are available, acquired with ESO's VLT equipped with FORS 1 (66.D-0128). The three B-band observations were added, and the photometry was calibrated with the USNO B1 catalogue due to missing archival calibrations. At the position of the two interesting sources, there are no optical sources down to the $\approx 26$ mag limit of the FORS $1 \mathrm{~B}$-band observations. Thus, the NIR-sources could not be excluded as possible substellar companions on the basis of their $B-H$ colour. Only a second-epoch observation, as soon as the proper motion of the NS itself is constrained, could clarify possible companionship.

RX J0806.4-4123 is one of the faintest of the seven XTINSs (e.g. Haberl et al. 2004). Neither proper motion nor distance were measured due to a still missing optical counterpart. The hydrogen column density fitted in the X-ray spectrum indicates a distance of $240 \pm 25$ pc taking into account the local inhomogeneously distributed ISM (Posselt et al. 2007). Possible substellar companions around RX J0806.4-4123 should have $H$-band magnitudes fainter than $18.6 \mathrm{mag}$ We find around 50 interesting NIR-sources within $5000 \mathrm{AU}$ down to $H=22.0$ mag that have a minimal SNR of three. We observed the crowded field in the $V$-band using ESO's NTT equipped with the $S$ uperb $S$ eeing Imager-2 (S US I2) in December 2004. The data reduction considered darks and flat fielding, bias subtraction was neglected. Astrometry for the optical observations was completed by fitting with the USNO B1 point source positions. The photometry was obtained by using a standard field observation reduced in the same way as the science field. The derived limit for $3 \sigma$ detections was $V=24.8 \mathrm{mag}$. A few NIR-sources could be excluded as possible substellar companions due to their low $V-H$-colour.
However, most of the interesting objects were not detected in the $V$-band. All the final 45 objects will have to be investigated after observing a second-epoch of the field.

RXS J214303.7+065419, also called RBS 1774, is a XTINS. To date, we do not know of an optical counterpart down to $r^{\prime}=25.7$ mag (Rea et al. 2007). The most recent X-ray position measured using Chandra has an uncertainty radius of 0.6" (Rea et al. 2007). Here, we assume $300 \mathrm{pc}$ to be a lower distance limit and an age similar to that of RX J1856.6-3754. Substellar companions are then expected to be fainter than $H=19$ mag. There are nine interesting objects within $5000 \mathrm{AU}$ of the neutron star position. Due to the availability of only three relatively bright and clustered 2MASS point sources, the photometric error of the sources is around $1 \mathrm{mag}$, especially for faint objects. The astrometric calibration was improved by using additional USNO B1 point source positions. The archival ESO EMMI R-band observations by Zampieri et al. (2001) have a limit of $R=23$ mag. $E M M I$ detected objects, some also present as USNO B1 point sources, provided the $R-H$ colour for the NIR-sources. For five NIR-sources, only the limit of $R=23$ mag could be applied, and thus no statement of a colour in favour of substellar companionship is possible. The four sources of known $R$-band magnitude can be excluded as possible substellar companions due to their $R-H$ colour, even considering a colour magnitude error of $2 \mathrm{mag}$. Two of the sources are elongated and are probably galaxies. Whether the remaining three sources are comoving objects or not can be ascertained only with the knowledge of the still unknown proper motion.

\subsection{H-band limits at the neutron-star positions}

In the following, we list the $H$-band limits derived from our observations. Since the main focus of our observations was to obtain relative astrometry, no standard stars were observed and sky conditions were not generally photometric. Furthermore, individual runs from different days have been combined to detect faint sources. Zeropoints (and their errors) obtained during the standard calbration plan by ESO were taken to derive an approximate photometric calibration. The zeropoints are from the same date as the individual runs (see Table A.1) and were averaged according to the combination of the runs. The StarFinder-Code was applied to the photometric measurements resulting in relatively small, nominal, magnitude errors. Due to the observing conditions (usually clear or thin cirrus) and the combinations of data from individual runs the error was larger. In Table 3, we report the $H$-band limits at the positions of the neutron stars as well as the determined minimal photometric errors. For a sufficient number (at least 5) of 2MASS sources with good quality flags in all bands, we compared our StarFinder magnitudes with those measured by 2 MASS for the respective sources. A correction to our magnitudes was then applied as well as its additional magnitude error (standard deviation of the found differences). For fields without sufficient 2MASS source numbers, an assumed magnitude error of $0.1 \mathrm{mag}(1 \sigma)$ was considered in addition to the StarFinder and zeropoint error. This reflects the estimated uncertainties in the sky conditions and the airmass correction for the respective fields. The average atmospheric extinction in the $H$-band at Paranal is $0.06 \mathrm{mag}^{2}$. We also list the Source Extractor magnitudes derived by completing only

\footnotetext{
2 http://www . eso.org/instruments/isaac/tools/ imaging_standards.html\#BB
} 
Table 3. $H$-band and mass limits obtained from the observations.

\begin{tabular}{lclccc}
\hline \hline Object & $\begin{array}{c}H_{\text {lim }} \pm 1 \sigma^{a} \\
{[\mathrm{mag}]}\end{array}$ & $\begin{array}{l}Z P^{b} \\
{[\mathrm{mag}]}\end{array}$ & $\begin{array}{c}S E-2 M A S S-\text { limits }^{c} \\
{[\mathrm{mag}]}\end{array}$ & $\begin{array}{c}\text { Lo Curto et al. (2007) } \\
{[\mathrm{mag}]}\end{array}$ & $\begin{array}{c}\text { Mass-limit }^{d} \\
\text { [Jupiter masses] }\end{array}$ \\
\hline PSR J0108-1431 & $21.42 \pm 0.16$ & 24.66 & $21.5 \pm 1.5$ & $\ldots$ & - \\
RX J0420.0-5022 & $21.84 \pm 0.16$ & 24.61 & $21.5 \pm 0.6$ & 21.7 & $\ldots$ \\
Geminga $^{e}$ & $22.66 \pm 0.14$ & $24.66^{\mathrm{e} 2}$ & $22.0 \pm 0.4$ & $\ldots$ & 12 \\
RX J0720.4-3125 $^{\mathrm{p}}$ & $23.07 \pm 0.11$ & $24.63^{\mathrm{ep} 2}$ & $22.3 \pm 0.4$ & 22.7 & $\ldots$ \\
RX J0806.4-4123 & $22.64 \pm 0.16$ & 24.59 & $22.6 \pm 0.4$ & 22.9 & 11.5 \\
RX J1856.6-3754 & $21.54 \pm 0.24$ & $24.65^{\mathrm{ep} 2}$ & $22.1 \pm 0.4$ & $\ldots$ & 42 \\
PSR J1932+1059 & $20.31 \pm 0.18$ & 23.49 & $\ldots$ & 22.1 & $\ldots$ \\
RBS 1774 & $22.04 \pm 0.16$ & 24.67 & $21.6 \pm 1.0$ & $\ldots$ \\
\hline
\end{tabular}

${ }^{a} H_{\text {lim }}$ indicates the faintest $H$-magnitude with a detection SNR of three measured close to the position of the neutron star; The $1 \sigma$ error is a result of the StarFinder photometric error, the zeropoint error, and an estimated/assumed error from comparison with $2 \mathrm{MASS} ;{ }^{b} Z P$ states the magnitude of the zeropoints, which were obtained in the frame of ESO's calibration plan; ${ }^{\text {ep2 }}$ indicates that two epochs have been used; ${ }^{c} S E-2 M A S S-l i m i t s$ lists the Source Extractor limits if using relative 2MASS photometry; ${ }^{d}$ The mass-limit is according to Sect. $5.1, \cdots$ notes that the found objects are interesting for a second epoch observation, - states the opposite; ${ }^{e}$ Geminga has been detected in a deeper HST F160W observation with roughly $24.1 \mathrm{mag}$ (Shibanov et al. 2006).

relative photometry with the available, preferably faint, 2MASS sources. We regard these magnitudes as less reliable since often only a few 2MASS sources were present (e.g. only two sources for the field of PSR J0108-1431) and not all have good quality flags. We therefore assigned a much higher magnitude error to these "SE 2MASS-limits". For some of our observations, Lo Curto et al. (2007) estimated $H$-band limits (by relative photometry with all 2MASS sources in the field), which are listed for comparison in Table 3. They stated a general magnitude error of 0.1 to 0.2 mag. Apart from RX J0720.4-3125, for which we measured a slightly fainter limit due to its second-epoch observation, our values correspond, within the error bars, to the results for 4 XTINSs investigated by Lo Curto et al. (2007).

If two epoch observations are available, it would be interesting if the combined image provides a deeper limit. For PSR $\mathrm{J} 1932+1059$ the NACO observation improves the constraints to the $H$-band limit since the bright source close to the pulsar was behind the coronograph. In the NACO field, sources are detected down to $21.3 \mathrm{mag}$. Since photons from the bright star are present close to the position of the NS, this might hinder detection of very faint objects. We therefore consider the $H$ magnitude of the faintest source to be our limit, which has a similar angular separation from the bright source as the pulsar. Thus, the $H$-band limit for PSR J1932+1059 is $20.31 \pm 0.18 \mathrm{mag}$.

When data for two ISAAC epochs (with similar observing conditions) were present, we combined both to test for a deeper limit for Geminga and RX J0720.4-3125. The zeropoints were averaged for the two epochs and StarFinder measurements were refined with high-quality-2MASS point sources as described above. In case of RX J1856.6-3754, the SOFI image was transformed to the ISAAC reference frame and both images were averaged. The StarFinder magnitudes were first calibrated approximately using the ISAAC zeropoint and then refined by using in addition 2MASS data. In Table 3, we list the results of the combined observations. In Fig. 6, the images around the NS position are shown, and in cases for which second-epoch data are available, the combined image is chosen. The field of PSR J1932+1059 is shown in Fig. 2. For RX J0806.4-4123, one might suspect a very faint source within the $r=1.5^{\prime \prime}$ circle. However, this does not represent an $3 \sigma$ detection ( $3 \sigma \mathrm{H}$-band limit is $22.64 \pm 0.16 \mathrm{mag}$ ). Deeper observations would be invaluable here although the field of RX J0806.4-4123 is very crowded and the probability of background objects is high.

\section{Discussion}

We have analysed multiple-epoch $H$-band observations of four isolated neutron stars and detected no comoving object with our data. The derived limits in the frame of the B97 calculations are 12, 15, 11, and 42 Jupiter masses for Geminga, RX J0720.43125, RX J1856.6-3754, and PSR J1932+1059, assuming ages of 1, 1, 1, and 3.1 Myr and distances of 250,361, 167, and $361 \mathrm{pc}$, respectively. The small size of the sample of investigated objects enables only crude statistical conclusions to be reached. A new query at the most recent ATNF-pulsar data base (e.g., Manchester et al. 2005 3 ) for pulsars of ages below $100 \mathrm{Myr}$ and distances below $400 \mathrm{pc}$, found ten NSs (including Geminga, and the XTINSs RX J0720.4-3125 and RX J1856.63754). Listed among them is the double-pulsar PSR J07373039. However, we excluded this system because the distance stated in the corresponding reference (Kramer et al. 2006) ranges from 200 to $1000 \mathrm{pc}$, and its inclusion would have involved a merging of statistics for double NS systems with isolated NSs. We considered two XTINSs in addition to RX J0720.4-3125 and RX J1856.6-3754 to represent the upper limit to the number of XTINSs within $400 \mathrm{pc}$. This upper limit was chosen because first, the second brightest XTINS, RX J0720.4-3125, has a distance of $361 \mathrm{pc}$, and second, we accounted for indications from the absorbing hydrogen column density and ISM models of distances to the fainter XTINSs (e.g. Posselt et al. 2007), Thus, we consider 11 NSs within $400 \mathrm{pc}$ as being sufficiently young $(<100 \mathrm{Myr})$ to exhibit comoving companions with our technique. We note that we do not include Rotational Radio Transients, since the currently-identified sources are inferred to be at distances between of 2 to $5 \mathrm{kpc}$ (McLaughlin et al. 2006). The probability, $P$, of achieving no detections in a subsample of 11 NSs with an (unknown) likelihood, $L_{\mathrm{Com}}$, of having substellar companions, is equivalent to an hypergeometric distribution probability. $P$ can be calculated according to the conventional equations of the hypergeometric distribution for different likelihoods $L_{\mathrm{Com}}$. Given 11 sources, a zero detection rate of brown dwarfs among four NSs results in values of $P=2 \%, 21 \%$, or $64 \%$, if $L_{\text {Com }}$ is $55 \%, 27 \%$, or $9 \%$, respectively. For planets heavier than 15 Jupiter masses, the zero detection rate of substellar companions among three NSs results in $P=6 \%, 34 \%$, or $73 \%$, if $L_{\mathrm{Com}}$ is $55 \%, 27 \%$, or $9 \%$, respectively. It is likely

\footnotetext{
${ }^{3}$ http://www.atnf.csiro.au/research/pulsar/psrcat
} 


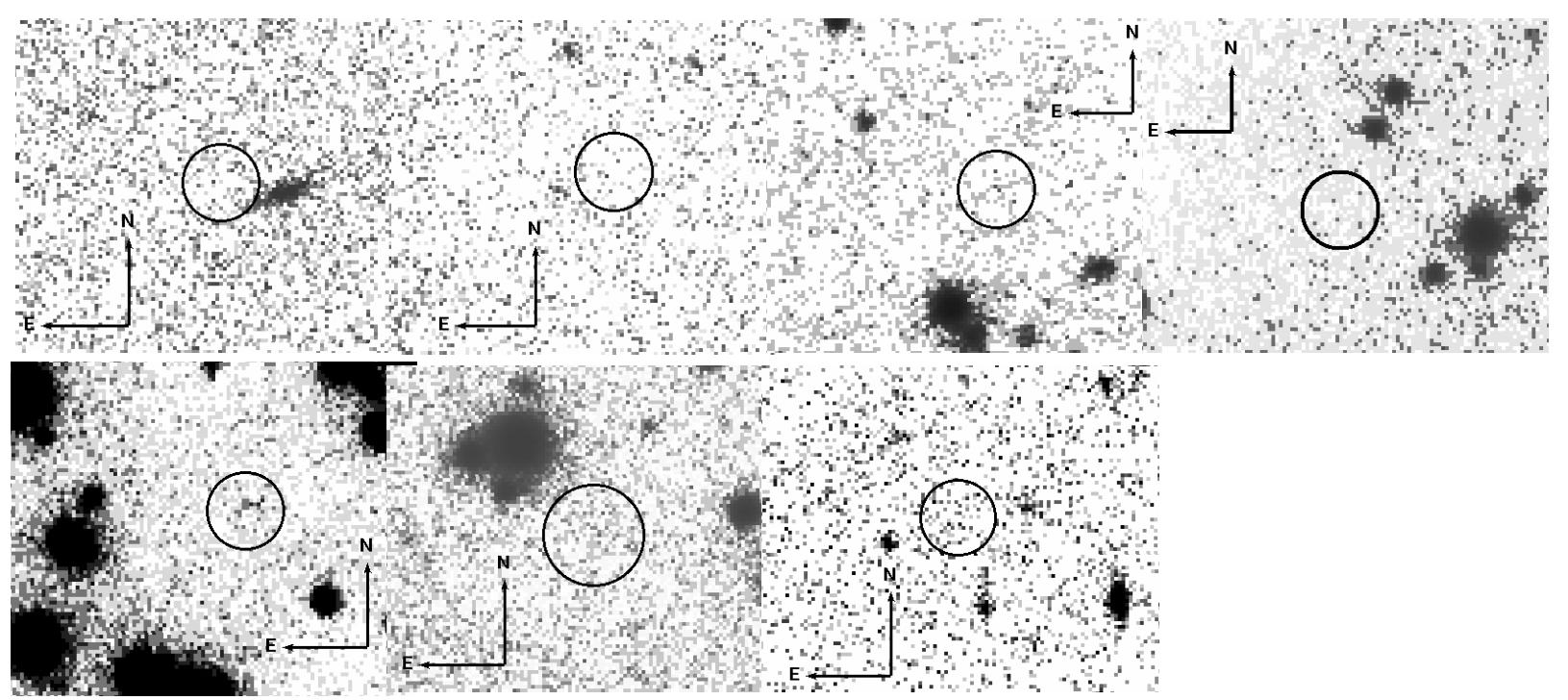

Fig. 6. Neutron star detection limits in the $H$-band. Moving left to right, top to bottom the $H$-band fields of the following neutron stars are shown: first row: PSR J0108-1431, RX J0420.0-5022, Geminga, RX J0720.4-3125; second row: RX J0806.4-4123, RX J1856.6-3754, RBS 1774. Except one each circle has a radius of $1.5^{\prime \prime}$. The radius for the circle around the position of RX J1856.6-3754 has a radius of $2^{\prime \prime}$. The box sizes are roughly $16^{\prime \prime} \times 13^{\prime \prime}$. See also text.

that the young population of isolated NSs has a similar substellar companion fraction as older isolated NSs. Strictly speaking, the numbers presented above cover only the case of formation of substellar companions after the supernova since we approximated the oldest age of possible substellar companions with the NS age. However, the SN survivor's rate is likely to be even smaller. We conclude that brown dwarfs and giant gas planets heavier than 15 Jupiter masses are rare among isolated NSs, for the following possible reasons: (1.) These substellar companions did not survive the destructive past of the NS; (2.) They are too massive to be formed from a supernova fallback disc; (3.) Massive giant planets ( $>10 M_{\text {Jupiter }}$ ) are rare, at least among well-studied, low-mass stars. Fewer than $5 \%$ of the known planets are such objects (The Extrasolar Planets Encyclopaedia by Schneider, as of March 2008 ${ }^{4}$ ); (4.) Only a few of the massive, stellar progenitors host giant planets. There are indications that the frequency of finding substellar companions decreases with stellar mass. Considering low-mass $\left(<5 M_{\odot}\right)$ stars, Kennedy \& Kenyon (2008) found, for example, a peak in the probability of having at least one gas-giant companion for stellar host masses $\sim 3 M_{\odot}$. While from massive-star formation sites, one knows of discs around protostars with masses less than $\sim 20 M_{\odot}$ (Cesaroni et al. 2007), these discs are expected to have a shorter lifetime than those around low-mass stars, and this may reduce the formation rate of massive substellar companions. There is currently no planet known around a star more massive then $4.5 M_{\odot}$ (The Extrasolar Planets Encyclopaedia by Schneider, as of March 2008), which may be, however, partly due to instrumental limitations (e.g. brightness contrast); (5.) Substellar companions may be fainter than calculated in B97. Comparing with other theoretical models based on non-gray atmospheres, the B97 effective temperatures are hotter (Baraffe et al. 2002). Thus, the true $H$-magnitudes may be lower than calculated by B97.

Considering our result in the light of searches for substellar companions around white dwarfs, it is interesting to note that substellar companions also appear to be uncommon around white dwarfs: until now only three L-type companions among well over 1000 white dwarf targets are known (Farihi et al. 2008

4 The Extrasolar Planets Encyclopaedia: http://exoplanet.eu/ and references therein) and one suspected planet $\left(\sim 2 M_{\text {Jupiter }}\right)$ around a pulsating white dwarf (Mullally et al. 2008) has yet to be confirmed.

We finally include a short note about RX J0720.4-3125: Haberl et al. (2006) proposed that free precession was responsible for the $\sim 7$ years variations in the pulsed X-ray spectra, although this is still a subject under discussion (van Kerkwijk et al. 2007). In the context of our work, it is interesting to note, why forced precession, e.g., by a substellar companion, is impossible. For example, Liu et al. (2007) discussed forced precession by a planet for PSR B1828-11. In the case of RX J0720.4-3125, we can apply the same formula as Liu et al. (2007) to approximate the orbital radius needed for planets lighter than 15 Jupiter masses to cause the precession observed. The largest orbital radius these planets could have, would be $1 \times 10^{-5} \mathrm{AU}$ (for zero inclination, NS distortion of $4 \times 10^{-8}$ ). However, this would be below the smallest Roche limit for a (normal) NS (radius of $10 \mathrm{~km}$, density of $10^{16} \mathrm{~kg} \mathrm{~m}^{-3}$ ), both for planets of densities similar to Earth, corresponding to a Roche limit of $2 \times 10^{-3} \mathrm{AU}$; and for planets with densities similar to Jupiter, resulting in a Roche limit of $3 \times 10^{-3} \mathrm{AU}$. Thus, even if there were planets lighter than 15 Jupiter masses around RX J0720.4-3125, they could not generate $\mathrm{a} \sim 7$ years precession of the NS.

In agreement with expectations, no neutron star was detected in $H$-band data. The NIR limits provide only weak contraints on the overall NS spectra given the tighter constraints from deep optical observations. This result agrees with that of the independent investigation for XTINSs by Lo Curto et al. (2007).

Acknowledgements. We thank A. Seifahrt for extensive discussions about source detection algorithm accuracy, L. Tacconi-Garman for suggestions regarding the $\mathrm{NACO}$-observations, as well as the service astronomers at ESO for observing our sources. Furthermore, we thank also P. Draper and E. Diolaiti for answering questions regarding GAIA and StarFinder. We thank the anonymous referee for careful reading and constructive comments which helped improving the quality of this article. This research has made use of SAOImage DS9, developed by SAO; the SIMBAD and VizieR databases, operated at CDS, Strasbourg, France; and SAO/NASA's Astrophysics Data System Bibliographic Services. 
Table A.1. List of our $H$-band observations.

\begin{tabular}{|c|c|c|c|c|c|c|c|}
\hline Object & Instrument & $\begin{array}{c}\text { Exposure } \\
{[\mathrm{s}]}\end{array}$ & $\begin{array}{c}\text { Nights } \\
\text { yyyy-mm-dd }\end{array}$ & Airmass & $\begin{array}{l}\text { DIMM Seeing } \\
\text { [arcsec] }\end{array}$ & Sky & $\begin{array}{c}\text { Included? } \\
\text { Yes / No }\end{array}$ \\
\hline \multirow{2}{*}{$\begin{array}{l}\text { PSR J0108-1431 } \\
\end{array}$} & VLT ISAAC & 1200 & $2003-06-23$ & 1.1 & 0.7 & photometric & Y-mod \\
\hline & VLT ISAAC & 1380 & 2003-08-07 & 1.0 & 0.8 & photometric & Y-mod \\
\hline \multirow[t]{3}{*}{ RX J0420.0-5022 } & VLT ISAAC & 1980 & 2004-01-10 & 1.3 & 0.6 & clouds & $\mathrm{N}$ \\
\hline & VLT ISAAC & 1980 & 2004-01-13 & 1.7 & 0.9 & photometric & Y-mod \\
\hline & VLT ISAAC & 1980 & 2003-11-13 & 1.4 & 0.8 & clear & Y \\
\hline \multirow[t]{3}{*}{ Geminga } & VLT ISAAC & 1980 & 2004-01-02 & 1.6 & 0.9 & photometric & $\mathrm{Y}$ \\
\hline & VLT ISAAC & 900 & 2003-12-30 & 1.4 & 0.6 & cirrus & $\mathrm{Y}$ \\
\hline & VLT ISAAC & 1980 & $2003-11-15$ & 1.4 & 0.5 & clear & Y \\
\hline \multirow[t]{3}{*}{ RX J0720.4-3125 } & VLT ISAAC & 1980 & 2004-01-08 & 1.0 & 0.5 & cirrus & $\mathrm{Y}$ \\
\hline & VLT ISAAC & 1980 & 2004-01-11 & 1.0 & 0.7 & clear & $\mathrm{Y}$ \\
\hline & VLT ISAAC & 1980 & 2003-12-04 & 1.0 & 0.8 & photometric & $\mathrm{Y}$ \\
\hline \multirow[t]{3}{*}{ RX J0806.4-4123 } & VLT ISAAC & 1980 & 2004-12-21 & 1.1 & 0.7 & photometric & $\mathrm{Y}$ \\
\hline & VLT ISAAC & 1980 & 2004-12-21 & 1.1 & 0.6 & photometric & $\mathrm{Y}$ \\
\hline & VLT ISAAC & 1980 & 2004-11-25 & 1.1 & 0.7 & clear & Y \\
\hline \multirow[t]{2}{*}{ RX J1856.6-3754 } & VLT ISAAC & 1380 & $2003-05-22$ & 1.1 & 0.7 & clear & $\mathrm{Y}$ \\
\hline & VLT ISAAC & 1380 & $2003-05-22$ & 1.1 & 0.7 & clear & $\mathrm{Y}$ \\
\hline \multirow[t]{3}{*}{ PSR J1932+1059 } & VLT ISAAC & 1380 & 2003-06-05 & 1.3 & 0.6 & clear & Y \\
\hline & VLT ISAAC & 1380 & 2003-06-04 & 1.3 & 0.8 & clear & Y-mod \\
\hline & VLT ISAAC & 1380 & 2003-06-17 & 1.7 & 0.7 & clear & $\mathrm{Y}$ \\
\hline \multirow[t]{2}{*}{ PSR J2124-3358 } & VLT ISAAC & 1380 & 2003-06-05 & 1.0 & 0.5 & clear & Y \\
\hline & VLT ISAAC & 1380 & $2003-05-22$ & 1.0 & 0.7 & clear & Y-mod \\
\hline \multirow[t]{2}{*}{ RBS 1774} & VLT ISAAC & 1200 & 2003-06-06 & 1.2 & 0.6 & clear & $\mathrm{Y}$ \\
\hline & VLT ISAAC & 1380 & 2003-06-06 & 1.2 & 0.5 & clear & $\mathrm{Y}$ \\
\hline \multirow{5}{*}{$\begin{array}{l}\text { Second epoch o } \\
\text { Geminga }\end{array}$} & rvations & & & & & & \\
\hline & VLT ISAAC & 2040 & 2007-02-08 & 1.5 & 0.8 & clear & Y-mod \\
\hline & VLT ISAAC & 2040 & 2007-02-08 & 1.7 & 0.7 & clear & Y-mod \\
\hline & VLT ISAAC & 2040 & $2007-01-26$ & 1.7 & 0.5 & photometric & Y \\
\hline & VLT ISAAC & 2040 & 2007-01-27 & 1.4 & 0.7 & photometric & Y \\
\hline \multirow[t]{4}{*}{ RX J0720.4-3125 } & VLT ISAAC & 1980 & 2006-12-27 & 1.3 & 0.8 & clear & $\mathrm{Y}$ \\
\hline & VLT ISAAC & 3420 & $2006-12-27$ & 1.1 & 0.9 & clear & Y-mod \\
\hline & VLT ISAAC & 2040 & $2006-12-25$ & 1.1 & 0.8 & clear & Y \\
\hline & VLT ISAAC & 2040 & $2006-12-25$ & 1.1 & 0.9 & clear & Y \\
\hline \multirow[t]{3}{*}{ RX J1856.6-3754 } & NTT SOFI & 24240 & 2006-05-09 & 1.3 & 0.8 & clear & Y-mod \\
\hline & NTT SOFI & 15960 & $2006-05-10$ & 1.4 & 0.7 & cirrus & Y-mod \\
\hline & NTT SOFI & 9120 & 2006-05-11 & 1.5 & 0.8 & cloudy & Y-mod \\
\hline \multirow[t]{3}{*}{ PSR J1932+1059 } & NTT SOFI & 7200 & 2006-05-10 & 1.4 & 0.7 & cirrus & Y-mod \\
\hline & NTT SOFI & 14400 & 2006-05-11 & 1.6 & 0.8 & cloudy & Y-mod \\
\hline & VLT $N A C O$ & 1360 & $2007-05-02$ & 1.3 & 0.6 & clear & Y-mod \\
\hline
\end{tabular}

Notes: the night parameters of each observation are averaged. Note that the DIMM seeing is the standard ESO $V$-band value. The Near Infrared seeing is usually of higher quality than at least 0.1 arcsec. In the column "Included ?" we stated whether we considered this observation run or did not use it, e.g., because of known technical problems or bad weather during the observations. Furthermore, it is noted by "Y-mod" if individual files were excluded from the observational run, e.g., due to exceptional bad seeing. For completeness, we note that five neutron star fields were already observed in December 2000 using ESO's NTT equipped with the SOFI instrument in the H-band (66.D-0135). However, compared to the later VLT ISAAC observations, the SOFI images were generally less deep and suffered from worse seeing conditions. We do not list the NTT SOFI observations from 2000 here.

\section{Appendix A: Observations}

The VLT NAos COnica (NACO) coronographic observations of PSR J1932+1059 were acquired by the S54 camera and the opaque $1.4^{\prime \prime}$ corongraph. As a reference star for the AO correction, the bright $(V=13.4)$ star GSC2 N0232103780 was selected, located $5^{\prime \prime}$ away from PSR J1932+1059. A jitter box of $20^{\prime \prime}$, coronographic individual exposures of $60 \mathrm{~s}$, sky individual exposures of $0.3454 \mathrm{~s}, 4$ "object" offset positions, and 6 "sky" offset positions were additional parameters of this NACO run. The jitter engine of Eclipse was applied for data reduction considering the corresponding dark and lamp flat, 28 individually selected objects for the image-stacking algorithm, and separate object and sky files.

Due to the relatively small number of images, the leakingin photons of the bright object outside the field of view, and the coronograph wire-construction, the resulting image (Fig. 2) shows several faint holes and artefacts, which may result in false source detections. We inspected each detected source and excluded obvious artefacts from further analysis. Since we were not interested in highly accurate photometry but in (relative) astrometry, the image quality served its intended purpose.

\section{Appendix B: Further details of methods}

\section{B.1. Other source detection algorithms}

For the relative astrometry, we considered two different approaches. First, we applied GAIA (Draper et al. 2007) for object detection provided that a signal-to-noise ratio (SNR) of at least three was reached. GAIA uses the Source Extractor provided by Bertin \& Arnouts (1996). In this program, which was designed with barycenter-detection of galaxies in mind, one can choose several detection parameters such as the type of filters to use (e.g. Gaussian), or how overlapping sources should be treated. 
We tested several sets of parameters and applied the apparently most suitable ones individually for each observational field. We then estimated the $H$-band magnitude by completing calibrations with (preferably faint) 2MASS PSC sources. The achieved SNR for a 22 mag object was around three, and magnitude errors were around $0.4 \mathrm{mag}$. The positional accuracy for faint sources stated by the Source Extractor was often surprisingly good (of higher precision than one tenth of pixel for faint sources), which does not appear realistic. We checked the positional accuracy reached by $2 \mathrm{D}$-Gaussfits using IDL. For the interesting - usually faint - objects, three times the mean positional uncertainty was $3 \sigma \approx 80$ mas, but could also exceptionally reach 230 mas for the faintest sources. The pixel scale of $I S A A C$ was $\approx 147$ mas. Brighter objects had usually more accurately determined positions. While the 2D-Gaussfits were useful for illustrating the possible maximum positional errors, they were unsuitable for overlapping sources. Therefore, as a final approach for the relative astrometry we applied StarFinder by Diolaiti et al. (2000b) of known accuracy as noted in Sect. 4. For bright $H$-band objects, the $3 \sigma$ error of the pixel shift is around one hundredth of a pixel, while, for the faintest objects, it reaches one pixel. We compared the Source Extractor / GAIA source list with the StarFinder source list for a performance check. StarFinder was usually able to find at least as many or more sources than the Source Extractor of GAIA, and only failed for galaxies that were excluded anyway from further analysis.

\section{B.2. Procedure in the case of first epoch only observations}

If no second-epoch observation was obtained, e.g., due to still unknown proper motion, we used the following procedure to identify at least potentially interesting objects for follow-up observations. Orbital separations of at least 5000 AU were inspected for NIR sources fainter than the magnitude obtained by the luminosity-magnitude relation and considering B97 at the brown-dwarf upper-mass limit. NS distances listed in Table 1 were used to determine the 5000 AU circle around the NS position. The relatively wide separations take into account the discovered distant substellar companions at roughly $4000 \mathrm{AU}$ around main-sequence stars (see e.g. Table 3 of Burgasser et al. 2005 , for brown-dwarf companions) and possible widening of orbits due to the mass loss of the primary during the supernova according to Kepler's laws.

The faint sources detected by GAIA were cross-correlated with sources in available optical observations to obtain opticalNIR-colours. This was usually completed with the help of archival or kindly provided optical observations. Many firstepoch-objects could be rejected as possible substellar companion candidates if these colours were typical of normal stars according to Kenyon \& Hartmann (1995). The given optical and optical-NIR-colours by Kenyon \& Hartmann (1995) go down to spectral type M6. As an example, one would expect the $R-H$ colour to be lower than 5 mag for normal stars down to M6 V, while L and T dwarfs often have higher values of around 6 mag. This can be seen in Fig. 1 of Kirkpatrick et al. (1999) for the $R-K$ colour (the $H-K$ colours are around 0.6 mag for most L and T dwarfs, see e.g. Fig. 14 of Kirkpatrick et al. 1999). However, we could not exclude ultra-cool M-dwarfs (M8) at this stage.

We note the use of a conventional magnitude error of usually $1 \mathrm{mag}$ up to an exceptional 2 mag for the optical-NIR-colours, considering variations in the magnitudes of the reference optical observations as well as errors in the magnitude estimation of the

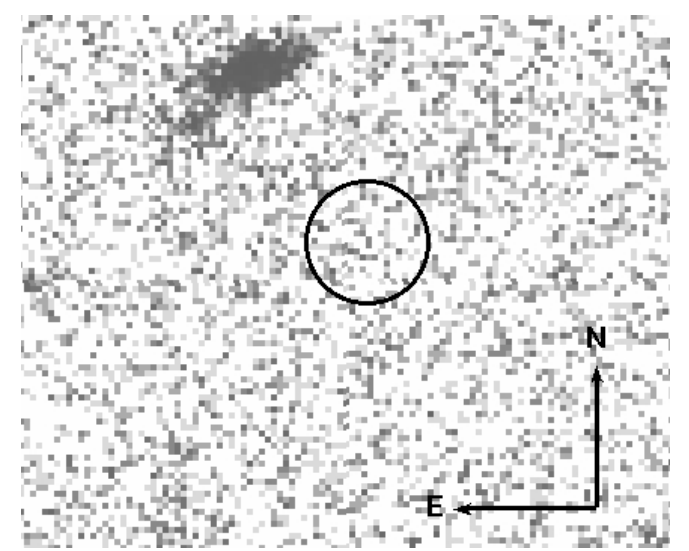

Fig. C.1. Detection limit in the $H$-band for PSR J2124-3358 The radius for the circle around the position of the NS has a radius of $1.5^{\prime \prime}$. The box size is roughly $16^{\prime \prime} \times 13^{\prime \prime}$.

NIR-objects. With this, all potentially interesting sources should be included.

\section{Appendix C: PSR J2124-3358}

PSR J2124-3358 is a millisecond radio pulsar. The currently upto-date ATNF pulsar catalogue (Manchester et al. 2005) indicates an age of $5.86 \times 10^{9}$ years, which is considerably older than we assessed when selecting this source some years ago from Taylor et al. (1993) in its latest online version (no age for PSR J2124-3358 noted). At this age and a distance of $270 \mathrm{pc}$, cooling substellar companions would have magnitudes fainter than $H=24.9 \mathrm{mag}$, following Burrows et al. (1997). Our ISAAC $H$-band observations are insufficiently deep to detect such objects. We derived the NIR limit at the position of the NS, which is $H_{\lim } \pm 1 \sigma=21.22 \pm 0.16 \mathrm{mag}$; the area around the NS is shown in Fig. C.1.

\section{References}

Baraffe, I., Chabrier, G., Allard, F., \& Hauschildt, P. H. 1998, A\&A, 337, 403 Baraffe, I., Chabrier, G., Allard, F., \& Hauschildt, P. H. 2002, A\&A, 382, 563 Bertin, E. 2006, in Astronomical Data Analysis Software and Systems XV, ed. C. Gabriel, C. Arviset, D. Ponz, \& S. Enrique, ASP Conf. Ser., 351, 112 Bertin, E., \& Arnouts, S. 1996, A\&AS, 117, 393

Bignami, G. F., \& Caraveo, P. A. 1996, ARA\&A, 34, 331

Burgasser, A. J., Kirkpatrick, J. D., \& Lowrance, P. J. 2005, AJ, 129, 2849 Burrows, A., Marley, M., Hubbard, W. B., et al. 1997, ApJ, 491, 856 Caraveo, P. A., Bignami, G. F., Mignani, R., \& Taff, L. G. 1996, ApJ, 461, L91 Caraveo, P. A., Lattanzi, M. G., Massone, G., et al. 1998, A\&A, 329, L1

Cesaroni, R., Galli, D., Lodato, G., Walmsley, C. M., \& Zhang, Q. 2007, in Protostars and Planets V, ed. B. Reipurth, D. Jewitt, \& K. Keil, 197 Chatterjee, S., Cordes, J. M., Vlemmings, W. H. T., et al. 2004, ApJ, 604, 339 Christou, J. C., Pugliese, G., Köhler, R., \& Drummond, J. D. 2004, PASP, 116, 734

D’Amico, N., Stappers, B. W., Bailes, M., et al. 1998, MNRAS, 297, 28

Devillard, N. 1999, in Astronomical Data Analysis Software and Systems VIII, ed. D. M. Mehringer, R. L. Plante, \& D. A. Roberts, ASP Conf. Ser., 172, 333 Devillard, N. 2001, in Astronomical Data Analysis Software and Systems X, ed. F. R. Harnden, Jr., F. A. Primini, \& H. E. Payne, ASP Conf. Ser., 238, 525 Diolaiti, E., Bendinelli, O., Bonaccini, D., et al. 2000a, A\&AS, 147, 335 Diolaiti, E., Bendinelli, O., Bonaccini, D., et al. 2000b, in Adaptive Optical Systems Technology, ed. P. L. Wizinowich, Proc. SPIE, 4007, 879

Draper, P., Gray, N. D. S. B., \& Taylor, M. 2007, GAIA - Graphical Astronomy and Image Analysis Tool Starlink User Note 214 http://www.starlink.ac.uk/gaia

Faherty, J., Walter, F. M., \& Anderson, J. 2007, Ap\&SS, 308, 225 Farihi, J., Burleigh, M. R., \& Hoard, D. W. 2008, ApJ, 674, 421 Gaensler, B. M., Jones, D. H., \& Stappers, B. W. 2002, ApJ, 580, L137 Golimowski, D. A., Leggett, S. K., Marley, M. S., et al. 2004, AJ, 127, 3516 
Haberl, F. 2004, Adv. Space Res., 33, 638

Haberl, F., Motch, C., Zavlin, V. E., et al. 2004, A\&A, 424, 635

Haberl, F., Turolla, R., de Vries, C. P., et al. 2006, A\&A, 451, L17

Hobbs, G., Manchester, R., Teoh, A., \& Hobbs, M. 2004, in IAU Symp., ed. F. Camilo, \& B. M. Gaensler, 139

Hotan, A. W., Bailes, M., \& Ord, S. M. 2006, MNRAS, 369, 1502

Jonker, P. G., van der Klis, M., \& Groot, P. J. 2003, MNRAS, 339, 663

Kaplan, D. L. 2008, in 40 YEARS OF PULSARS: Millisecond Pulsars, Magnetars and More, AIP Conf. Ser., 968, 129

Kaplan, D. L., van Kerkwijk, M. H., \& Anderson, J. 2002, ApJ, 571, 447

Kaplan, D. L., van Kerkwijk, M. H., \& Anderson, J. 2007, ApJ, 660, 1428

Kennedy, G. M., \& Kenyon, S. J. 2008, ApJ, 673, 502

Kenyon, S. J., \& Hartmann, L. 1995, ApJS, 101, 117

Kirkpatrick, J. D., Reid, I. N., Liebert, J., et al. 1999, ApJ, 519, 802

Konacki, M., \& Wolszczan, A. 2003, ApJ, 591, L147

Kramer, M., Stairs, I. H., Manchester, R. N., et al. 2006, Science, 314, 97

Liu, K., Yue, Y. L., \& Xu, R. X. 2007, MNRAS, 381, L1

Lo Curto, G., Mignani, R. P., Perna, R., \& Israel, G. L. 2007, A\&A, 473, 539

Lorimer, D. R. \& Kramer, M. 2004, Handbook of Pulsar Astronomy Cambridge observing handbooks for research astronomers (Cambridge University Press), 4

Manchester, R. N., Hobbs, G. B., Teoh, A., \& Hobbs, M. 2005, AJ, 129, 1993

Manchester, R. N., \& Taylor, J. H. 1977, Pulsars (San Francisco: W. H. Freeman, c1977.), 36

McLaughlin, M. A., Lyne, A. G., Lorimer, D. R., et al. 2006, Nature, 439, 817

Mignani, R. P., Manchester, R. N., \& Pavlov, G. G. 2003, ApJ, 582, 978

Mink, D. J. 2002, in Astronomical Data Analysis Software and Systems VIII, ed. D. Mehringer, R. Plante, \& D. Roberts, ASP Conf. Ser., 172, 498

Monet, D. G., Levine, S. E., Canzian, B., et al. 2003, AJ, 125, 984
Motch, C., Pires, A. M., Haberl, F., Schwope, A., \& Zavlin, V. E. 2009, ArXiv e-prints 0901.1006

Motch, C., Zavlin, V. E., \& Haberl, F. 2003, A\&A, 408, 323

Mullally, F., Winget, D. E., Degennaro, S., et al. 2008, ArXiv e-prints, 801

Neuhäuser, R., \& Trümper, J. E. 1999, A\&A, 343, 151

Podsiadlowski, P. 1995, in Millisecond Pulsars, A Decade of Surprise, ed. A. S. Fruchter, M. Tavani, \& D. C. Backer, ASP Conf. Ser., 72, 411

Posselt, B., Popov, S. B., Haberl, F., et al. 2007, Ap\&SS, 308, 171

Rea, N., Torres, M. A. P., Jonker, P. G., et al. 2007, MNRAS, 379, 1484

Shibanov, Y. A., Zharikov, S. V., Komarova, V. N., et al. 2006, A\&A, 448, 313

Sigurdsson, S., Richer, H. B., Hansen, B. M., Stairs, I. H., \& Thorsett, S. E. 2003, Science, 301, 193

Sigurdsson, S., \& Thorsett, S. E. 2005, in Binary Radio Pulsars, ed. F. A. Rasio, \& I. H. Stairs, ASP Conf. Ser., 328, 213

Skrutskie, M. F., Cutri, R. M., Stiening, R., et al. 2006, AJ, 131, 1163

Taylor, J. H., Manchester, R. N., \& Lyne, A. G. 1993, ApJS, 88, 529

Tody, D. 1986, in Instrumentation in astronomy VI; Proceedings of the Meeting, Tucson, AZ, Mar. 4-8, Part 2 (A87-36376 15-35), Bellingham, WA, Society of Photo-Optical Instrumentation Engineers, ed. D. L. Crawford, 733

Tody, D. 1993, in Astronomical Data Analysis Software and Systems II, ed. R. J. Hanisch, R. J. V. Brissenden, \& J. Barnes, ASP Conf. Ser., 52, 173

Trümper, J. E., Burwitz, V., Haberl, F., \& Zavlin, V. E. 2004, Nucl. Phys. B Proc. Suppl., 132, 560

van Kerkwijk, M. H., Kaplan, D. L., Pavlov, G. G., \& Mori, K. 2007, ApJ, 659, L149

Walter, F. M., \& Lattimer, J. M. 2002, ApJ, 576, L145

Walter, F. M., \& Matthews, L. D. 1997, Nature, 389, 358

Wolszczan, A., \& Frail, D. A. 1992, Nature, 355, 145

Zampieri, L., Campana, S., Turolla, R., et al. 2001, A\&A, 378, L5 